\title{
Front Matter: Volume 10575
}

, "Front Matter: Volume 10575," Proc. SPIE 10575, Medical Imaging 2018: Computer-Aided Diagnosis, 1057501 (13 April 2018); doi: $10.1117 / 12.2315758$

SPIE. Event: SPIE Medical Imaging, 2018, Houston, Texas, United States 


\title{
PROGRESS IN BIOMEDICAL OPTICS AND IMAGING
}

\section{Medical Imaging 2018}

\section{Computer-Aided Diagnosis}

\author{
Nicholas Petrick \\ Kensaku Mori
}

\section{2-15 February 2018 \\ Houston, Texas, United States}

Sponsored by

SPIE

Co-sponsored by

DECTRIS Ltd. (Switzerland)

Cooperating Organizations

AAPM-American Association of Physicists in Medicine (United States)

IFCARS - International Foundation for Computer Assisted Radiology and Surgery (Germany)

MIPS-Medical Image Perception Society (United States)

RSNA-Radiological Society of North America (United States)

WMIS—World Molecular Imaging Society

Published by

SPIE

\section{Volume 10575}

Part One of Two Parts 
The papers in this volume were part of the technical conference cited on the cover and title page. Papers were selected and subject to review by the editors and conference program committee. Some conference presentations may not be available for publication. Additional papers and presentation recordings may be available online in the SPIE Digital Library at SPIEDigitalLibrary.org.

The papers reflect the work and thoughts of the authors and are published herein as submitted. The publisher is not responsible for the validity of the information or for any outcomes resulting from reliance thereon.

Please use the following format to cite material from these proceedings:

Author(s), "Title of Paper," in Medical Imaging 2018: Computer-Aided Diagnosis, edited by Nicholas Petrick, Kensaku Mori, Proceedings of SPIE Vol. 10575 (SPIE, Bellingham, WA, 2018) Sevendigit Article CID Number.

ISSN: 1605-7422

ISSN: 2410-9045 (electronic)

ISBN: 9781510616394

ISBN: 9781510616400 (electronic)

Published by

SPIE

P.O. Box 10, Bellingham, Washington $98227-0010$ USA

Telephone +1 3606763290 (Pacific Time) · Fax +1 3606471445

SPIE.org

Copyright (C) 2018, Society of Photo-Optical Instrumentation Engineers.

Copying of material in this book for internal or personal use, or for the internal or personal use of specific clients, beyond the fair use provisions granted by the U.S. Copyright Law is authorized by SPIE subject to payment of copying fees. The Transactional Reporting Service base fee for this volume is $\$ 18.00$ per article (or portion thereof), which should be paid directly to the Copyright Clearance Center (CCC), 222 Rosewood Drive, Danvers, MA 01923. Payment may also be made electronically through CCC Online at copyright.com. Other copying for republication, resale, advertising or promotion, or any form of systematic or multiple reproduction of any material in this book is prohibited except with permission in writing from the publisher. The CCC fee code is 1605$7422 / 18 / \$ 18.00$.

Printed in the United States of America.

Publication of record for individual papers is online in the SPIE Digital Library.

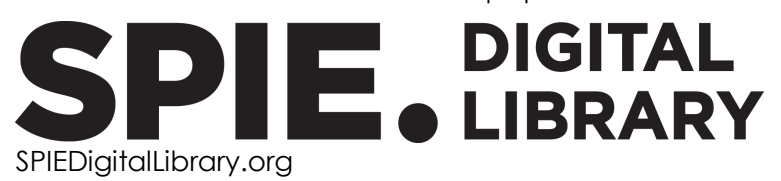

Paper Numbering: Proceedings of SPIE follow an e-First publication model. A unique citation identifier (CID) number is assigned to each article at the time of publication. Utilization of CIDs allows articles to be fully citable as soon as they are published online, and connects the same identifier to all online and print versions of the publication. SPIE uses a seven-digit CID article numbering system structured as follows:

- The first five digits correspond to the SPIE volume number.

- The last two digits indicate publication order within the volume using a Base 36 numbering system employing both numerals and letters. These two-number sets start with 00, 01, 02, 03, 04, 05, 06, 07, 08, 09, OA, OB ... OZ, followed by 10-1Z, 20-2Z, etc. The CID Number appears on each page of the manuscript. 


\title{
Contents
}

\author{
xiii Authors \\ xix Conference Committee \\ xxiii 2018 Medical Imaging Award Recipients
}

\section{Part One}

\section{SESSION 1 LUNG I AND LIVER}

1057502 Dense volumetric detection and segmentation of mediastinal lymph nodes in chest CT images [10575-1]

1057503 Early detection of lung cancer recurrence after stereotactic ablative radiation therapy: radiomics system design [10575-2]

1057504 Pneumothorax detection in chest radiographs using convolutional neural networks [10575-3]

1057505 Boosting CNN performance for lung texture classification using connected filtering [10575-4]

1057506 Automatic liver volume segmentation and fibrosis classification [10575-5]

SESSION 2 RADIOMICS

1057507 Association of high proliferation marker Ki-67 expression with DCEMR imaging features of breast: a large scale evaluation [10575-6]

1057508 Detecting mammographically occult cancer in women with dense breasts using Radon Cumulative Distribution Transform: a preliminary analysis [10575-7]

1057509 Deriving stable multi-parametric MRI radiomic signatures in the presence of inter-scanner variations: survival prediction of glioblastoma via imaging pattern analysis and machine learning techniques [10575-8]

10575 OA Robustness of radiomic breast features of benign lesions and luminal A cancers across MR magnet strengths [10575-9]

10575 OB Empirical evaluation of cross-site reproducibility in radiomic features for characterizing prostate MRI [10575-10]

10575 OC A deep learning classifier for prediction of pathological complete response to neoadjuvant chemotherapy from baseline breast DCE-MRI [10575-11] 
10575 OD Deep learning and texture-based semantic label fusion for brain tumor segmentation [10575-12]

10575 OE Quantifying the association between white matter integrity changes and subconcussive head impact exposure from a single season of youth and high school football using 3D convolutional neural networks [10575-13]

10575 OF Single season changes in resting state network power and the connectivity between regions distinguish head impact exposure level in high school and youth football players [10575-14]

10575 OG Gaussian processes with optimal kernel construction for neuro-degenerative clinical onset prediction [10575-15]

$10575 \mathrm{OH} \quad$ MRI textures as outcome predictor for Gamma Knife radiosurgery on vestibular schwannoma [10575-16]

10575 Ol ADMultilmg: a novel missing modality transfer learning based CAD system for diagnosis of $\mathrm{MCl}$ due to AD using incomplete multi-modality imaging data [10575-17]

10575 OJ Longitudinal connectome-based predictive modeling for REM sleep behavior disorder from structural brain connectivity [10575-18]

\section{SESSION 4 MUSCULOSKELETAL AND SKIN}

10575 OK Automated synovium segmentation in doppler ultrasound images for rheumatoid arthritis assessment [10575-19]

$10575 \mathrm{OL} \quad$ Automatic pedicles detection using convolutional neural network in a 3D spine reconstruction from biplanar radiographs [10575-20]

10575 OM Fully automated bone mineral density assessment from low-dose chest CT [10575-21]

10575 ON Segmentation of skin lesions in chronic graft versus host disease photographs with fully convolutional networks [10575-22]

1057500 Computer-aided detection of basal cell carcinoma through blood content analysis in dermoscopy images [10575-23]

10575 OP Improving performance of breast cancer risk prediction using a new CAD-based region segmentation scheme [10575-24]

$105750 Q \quad$ Cross-domain and multi-task transfer learning of deep convolutional neural network for breast cancer diagnosis in digital breast tomosynthesis [10575-25] 
10575 OR Improving classification with forced labeling of other related classes: application to prediction of upstaged ductal carcinoma in situ using mammographic features [10575-26]

10575 OS Deep learning in breast cancer risk assessment: evaluation of fine-tuned convolutional neural networks on a clinical dataset of FFDMs [10575-27]

10575 OT Transfer learning with convolutional neural networks for lesion classification on clinical breast tomosynthesis [10575-28]

10575 OU Breast tumor segmentation in DCE-MRI using fully convolutional networks with an application in radiogenomics [10575-29]

\section{SESSION 6 CARDIAC, VESSELS, AND NOVEL APPLICATIONS}

10575 OV A quality score for coronary artery tree extraction results [10575-30]

10575 OW Aortic root segmentation in 4D transesophageal echocardiography [10575-31]

10575 OX Automated assessment of aortic and main pulmonary arterial diameters using modelbased blood vessel segmentation for predicting chronic thromboembolic pulmonary hypertension in low-dose CT lung screening [10575-32]

$10575 \mathrm{OZ}$ Identification and functional characterization of HIV-associated neurocognitive disorders with large-scale Granger causality analysis on resting-state functional MRI [10575-34]

\section{SESSION 8 KEYNOTE AND EYE}

1057511 Automated segmentation of geographic atrophy using deep convolutional neural networks [10575-36]

1057512 An affordable and easy-to-use diagnostic method for keratoconus detection using a smartphone [10575-37]

\section{SESSION 9 COLON AND PROSTATE}

1057513 Detection of protruding lesion in wireless capsule endoscopy videos of small intestine [10575-38]

1057514 Radiomics analysis of DWI data to identify the rectal cancer patients qualified for local excision after neoadjuvant chemoradiotherapy [10575-39]

1057515 Development of a computer aided diagnosis model for prostate cancer classification on multi-parametric MRI [10575-40]

1057516 Cascade classification of endocytoscopic images of colorectal lesions for automated pathological diagnosis [10575-41] 
1057517 A new fractional order derivative based active contour model for colon wall segmentation [10575-42]

1057518 Detection of colorectal masses in CT colonography: application of deep residual networks for differentiating masses from normal colon anatomy [10575-43]

SESSION 10 HEAD AND NECK

1057519 Comparison of different deep learning approaches for parotid gland segmentation from CT images [10575-44]

10575 1A Detection of eardrum abnormalities using ensemble deep learning approaches [10575-45]

10575 1B A convolutional neural network for intracranial hemorrhage detection in non-contrast CT [10575-46]

10575 1C Deep 3D convolution neural network for CT brain hemorrhage classification [10575-47]

10575 ID Cerebral microbleed detection in traumatic brain injury patients using 3D convolutional neural networks [10575-48]

SESSION $11 \quad$ LUNG II

$10575 \mathrm{lE}$ Comparing deep learning models for population screening using chest radiography [10575-49]

10575 IF Deep-learning derived features for lung nodule classification with limited datasets [10575-50]

10575 IG Prognostic importance of pleural attachment status measured by pretreatment CT images in patients with stage IA lung adenocarcinoma: measurement of the ratio of the interface between nodule and neighboring pleura to nodule surface area [10575-51]

$10575 \mathrm{1H}$ Differentiating invasive and pre-invasive lung cancer by quantitative analysis of histopathologic images [10575-52]

1057511 Using YOLO based deep learning network for real time detection and localization of lung nodules from low dose CT scans [10575-53]

$105751 \mathrm{~J} \mathrm{Automated} \mathrm{volumetric} \mathrm{lung} \mathrm{segmentation} \mathrm{of} \mathrm{thoracic} \mathrm{CT} \mathrm{images} \mathrm{using} \mathrm{fully} \mathrm{convolutional}$ neural network [10575-54]

$105751 \mathrm{~K}$ Variations in algorithm implementation among quantitative texture analysis software packages [10575-55] 
10575 IL Towards quantitative imaging: stability of fully automated nodule segmentation across varied dose levels and reconstruction parameters in a low-dose CT screening patient cohort [10575-56]

$105751 \mathrm{M}$ Anomaly detection for medical images based on a one-class classification [10575-57]

10575 iN Quantitative CT analysis for the preoperative prediction of pathologic grade in pancreatic neuroendocrine tumors [10575-58]

1057510 Quantitative image feature variability amongst CT scanners with a controlled scan protocol [10575-59]

\section{SESSION 13 BRAIN II}

10575 IP A primitive study on unsupervised anomaly detection with an autoencoder in emergency head CT volumes [10575-60]

$105751 Q$ Artery and vein segmentation of the cerebral vasculature in 4D CT using a 3D fully convolutional neural network [10575-61]

10575 IR Detection of brain tumor margins using optical coherence tomography [10575-62]

10575 is Classification of brain MRI with big data and deep 3D convolutional neural networks [10575-63]

10575 IT Saliency U-Net: A regional saliency map-driven hybrid deep learning network for anomaly segmentation [10575-64]

$105751 \mathrm{U}$ Radiation-free quantification of head malformations in craniosynostosis patients from 3D photography [10575-65]

\section{SESSION 14 OTHER ORGANS}

10575 IV Bladder cancer treatment response assessment in CT urography using two-channel deeplearning network [10575-66]

$105751 \mathrm{~W}$ Automated detection and segmentation of follicles in 3D ultrasound for assisted reproduction [10575-67]

10575 1X Comparison of machine learned approaches for thyroid nodule characterization from shear wave elastography images [10575-68]

10575 IY Bladder cancer treatment response assessment with radiomic, clinical, and radiologist semantic features [10575-69]

$105751 \mathrm{Z}$ Automatic detection of kidney in 3D pediatric ultrasound images using deep neural networks [10575-70] 


\section{Part Two}

SESSION $15 \quad$ BREAST II

1057520 Generalization error analysis: deep convolutional neural network in mammography [10575-71]

1057521 Compression of deep convolutional neural network for computer-aided diagnosis of masses in digital breast tomosynthesis [10575-72]

1057522 ICADx: interpretable computer aided diagnosis of breast masses [10575-73]

1057523 Do pre-trained deep learning models improve computer-aided classification of digital mammograms? [10575-74]

1057524 Fully automated gynecomastia quantification from low-dose chest CT [10575-75]

1057525 Breast mass detection in mammography and tomosynthesis via fully convolutional network-based heatmap regression [10575-76]

POSTER SESSION: ABDOMINAL AND GASTROINTESTINAL

1057526 Detecting PHG frames in wireless capsule endoscopy video by integrating rough global dominate-color with fine local texture features [10575-77]

1057527 Automatic blood vessel based-liver segmentation using the portal phase abdominal CT [10575-78]

1057528 Deep convolutional neural network for the classification of hepatocellular carcinoma and intrahepatic cholangiocarcinoma [10575-79]

1057529 Computer-aided detection of bladder wall thickening in CT urography (CTU) (Cum Laude Poster Award) [10575-80]

10575 2A Histogram-based adaptive gray level scaling for texture feature classification of colorectal polyps [10575-81]

10575 2B Bladder cancer staging in CT urography: effect of stage labels on statistical modeling of a decision support system [10575-82]

10575 2C Performance evaluation of 2D and 3D deep learning approaches for automatic segmentation of multiple organs on CT images (Honorable Mention Poster Award) [10575-83]

10575 2D Urinary bladder cancer T-staging from T2-weighted MR images using an optimal biomarker approach [10575-141] 
$105752 \mathrm{E} \quad$ Automated volumetry of temporal horn of lateral ventricle for detection of Alzheimer's disease in CT scan [10575-84]

$105752 \mathrm{~F} \quad$ Exploring DeepMedic for the purpose of segmenting white matter hyperintensity lesions [10575-85]

$105752 \mathrm{G}$ Evaluation of a deep learning architecture for MR imaging prediction of ATRX in glioma patients [10575-86]

$105752 \mathrm{H} \quad$ Measurement of hard tissue density based on image density of intraoral radiograph [10575-88]

105752 Classifying magnetic resonance image modalities with convolutional neural networks [10575-89]

\section{POSTER SESSION: BREAST}

10575 2J Pectoral muscle segmentation in breast tomosynthesis with deep learning [10575-90]

$105752 \mathrm{~K} \quad$ Computer-aided classification of breast masses using contrast-enhanced digital mammograms [10575-91]

$105752 \mathrm{~L} \quad$ Applying a new unequally weighted feature fusion method to improve CAD performance of classifying breast lesions [10575-92]

10575 2M Recurrent neural networks for breast lesion classification based on DCE-MRIs [10575-93]

$105752 \mathrm{~N}$ Multi-resolution analysis using integrated microscopic configuration with local patterns for benign-malignant mass classification [10575-94]

1057520 Association between mammogram density and background parenchymal enhancement of breast MRI [10575-95]

$105752 P \quad$ Reduction of false-positives in a CAD scheme for automated detection of architectural distortion in digital mammography [10575-96]

$105752 \mathrm{~A}$ A fully automatic microcalcification detection approach based on deep convolution neural network [10575-97]

$105752 R \quad$ Learning better deep features for the prediction of occult invasive disease in ductal carcinoma in situ through transfer learning [10575-98]

1057525 Deformable image registration as a tool to improve survival prediction after neoadjuvant chemotherapy for breast cancer: results from the ACRIN 6657/I-SPY-1 trial [10575-99]

10575 2T Expert identification of visual primitives used by CNNs during mammogram classification [10575-100] 
$105752 \mathrm{U}$ Similarity estimation for reference image retrieval in mammograms using convolutional neural network [10575-101]

$105752 \mathrm{~V}$ Convolutional encoder-decoder for breast mass segmentation in digital breast tomosynthesis [10575-102]

10575 2W Deep learning-based features of breast MRI for prediction of occult invasive disease following a diagnosis of ductal carcinoma in situ: preliminary data [10575-103]

10575 2X Breast cancer molecular subtype classification using deep features: preliminary results [10575-104]

\section{POSTER SESSION: CARDIAC}

$105752 Y \quad$ Voxel-based plaque classification in coronary intravascular optical coherence tomography images using decision trees [10575-105]

$105752 Z$ Myocardial scar segmentation from magnetic resonance images using convolutional neural network [10575-106]

1057530 Convolutional neural networks for the detection of diseased hearts using $\mathrm{CT}$ images and left atrium patches [10575-107]

\section{POSTER SESSION: EYE}

1057531 Lesion detection in ultra-wide field retinal images for diabetic retinopathy diagnosis [10575-108]

POSTER SESSION: LUNG

1057533 3D GGO candidate extraction in lung CT images using multilevel thresholding on supervoxels [10575-110]

1057534 Opacity annotation of diffuse lung diseases using deep convolutional neural network with multi-channel information [10575-111]

1057535 Localization of lung fields in HRCT images using a deep convolution neural network [10575-112]

1057536 Deep neural network convolution (NNC) for three-class classification of diffuse lung disease opacities in high-resolution CT (HRCT): consolidation, ground-glass opacity (GGO), and normal opacity [10575-113]

1057537 A deep-learning based automatic pulmonary nodule detection system [10575-114]

1057538 An evaluation of consensus techniques for diagnostic interpretation [10575-115] 
1057539 Lung nodule detection from CT scans using 3D convolutional neural networks without candidate selection [10575-116]

10575 3A An automatically generated texture-based atlas of the lungs [10575-117]

10575 3B A coarse-to-fine approach for pericardial effusion localization and segmentation in chest CT scans [10575-118]

\section{POSTER SESSION: MUSCULOSKELETAL AND SKIN}

10575 3C Automated quasi-3D spine curvature quantification and classification [10575-119]

10575 3D Computer aided detection system for Osteoporosis using low dose thoracic 3D CT images [10575-120]

$105753 \mathrm{E}$ Automatic detection of anatomical regions in frontal x-ray images: comparing convolutional neural networks to random forest [10575-121]

POSTER SESSION: QUANTITATIVE

105753 F Applying a CAD-generated imaging marker to assess short-term breast cancer risk [10575-123]

$105753 G$ Asymmetry quantification from reflectance images of orthotic patients using structural similarity metrics [10575-124]

10575 3H Quantitative characterization of liver tumor radiodensity in CT images: a phantom study between two scanners [10575-125]

105753 Deep convolutional neural network for mammographic density segmentation [10575-126]

10575 3J Quantitative assessment for pneumoconiosis severity diagnosis using 3D CT images [10575-127]

10575 3K Quantitative analysis of adipose tissue on chest CT to predict primary graft dysfunction in lung transplant recipients: a novel optimal biomarker approach [10575-128]

$105753 \mathrm{~L}$ Impact of deep learning on the normalization of reconstruction kernel effects in imaging biomarker quantification: a pilot study in CT emphysema [10575-129]

$105753 \mathrm{M}$ Lung parenchymal analysis on dynamic MRI in thoracic insufficiency syndrome to assess changes following surgical intervention [10575-130]

$105753 \mathrm{~N}$ Applying a new mammographic imaging marker to predict breast cancer risk [10575-131] 
1057530 Quantitative CT based radiomics as predictor of resectability of pancreatic adenocarcinoma [10575-132]

$105753 \mathrm{P}$ Stability of deep features across CT scanners and field of view using a physical phantom [10575-133]

$105753 Q$ Temporal assessment of radiomic features on clinical mammography in a high-risk population [10575-134]

10575 3R Deep radiomic prediction with clinical predictors of the survival in patients with rheumatoid arthritis-associated interstitial lung diseases [10575-135]

1057535 Radiomic biomarkers from PET/CT multi-modality fusion images for the prediction of immunotherapy response in advanced non-small cell lung cancer patients [10575-136]

10575 3T Classification of brain tumors using texture based analysis of $\mathrm{T} 1$-post contrast MR scans in a preclinical model [10575-137]

$105753 \mathrm{U}$ Radiomics for ultrafast dynamic contrast-enhanced breast MRI in the diagnosis of breast cancer: a pilot study [10575-138]

$105753 \mathrm{~V}$ Reduction in training time of a deep learning model in detection of lesions in CT [10575-139]

$105753 \mathrm{~W}$ The effects of variations in parameters and algorithm choices on calculated radiomics feature values: initial investigations and comparisons to feature variability across $\mathrm{CT}$ image acquisition conditions [10575-140] 


\section{Authors}

Numbers in the index correspond to the last two digits of the seven-digit citation identifier (CID) article numbering system used in Proceedings of SPIE. The first five digits reflect the volume number. Base 36 numbering is employed for the last two digits and indicates the order of articles within the volume. Numbers start with 00, 01, 02, 03, 04, 05, 06, 07, 08, 09, OA, OB...0Z, followed by 10-12, 20-2Z, etc.

\author{
Abe, Hiroyuke, 3U \\ Abe, Osamu, $1 \mathrm{P}$ \\ Abidin, Anas Z., $\mathrm{OZ}$ \\ Aboutalib, Sarah S., 23 \\ Abuya, Joseph, $1 \mathrm{E}$ \\ Agarwala, Sunita, 35 \\ Aghaei, Faranak, 2K, 2O, 3F, 3N \\ Aitharaju, Sai, 15 \\ Akbari, Hamed, 09 \\ Alam, M., OD \\ Albadawy, Ehab, $2 x$ \\ Alderson, Philip, $1 \mathrm{E}$ \\ Alessio, Adam M., $1 \mathrm{X}$ \\ Alfano, R., 15 \\ Alfonso Burgos, N., 1D \\ Al-Hallaq, Hania, 1K \\ Allen, Peter J., $1 \mathrm{~N}$ \\ Alva, Ajjai, 1V, 1Y, 2B \\ Amitai, Michal, 06 \\ Anderson, Rachel, $3 \mathrm{U}$ \\ Antani, Sameer, $1 \mathrm{E}$ \\ Antropova, Natasha, 2M \\ Aokage, K., IG \\ Arbabshirani, Mohammad R., 1C \\ Arif, Wasim, 2N \\ Armato, Samuel G., $1 \mathrm{~K}$ \\ Ashizawa, Kazuto, 3J \\ Askarian, Amin, 12 \\ Askarian, Behnam, 12 \\ Aubert, Benjamin, OL \\ Azour, Lea, 24 \\ Bagheri, Mohammadhadi, 3B \\ Bai, Jianying, 13, 26 \\ Bakas, Spyridon, 09 \\ Bakhous, Christine, OL \\ Bal, Evgeny, 06 \\ Balagurunathan, Yoganand, 35 \\ Bauman, G. S., 15 \\ Bedrosian, Isabelle, $3 Q$ \\ Benzinger, Tammie, OG \\ Berg, Wendie A., 23 \\ Berman, Benjamin Paul, 3H \\ Beymer, David, 1J \\ Bhatia, Kanwal K., 02 \\ Biggs, Elijah, $1 Z$ \\ Bloch, B. Nicolas, OB \\ Blumenfeld, Aviel, 04 \\ Boucher, Marc-Antoine, $3 G$ \\ Braman, Nathaniel, OC
}

Brillet, Pierre-Yves, 05

Broersen, Alexander, OV

Brown, Matthew S., 1L, 3W

Buhmann, Joachim, is

Butman, John A., 2

Cai, Guanxiong, $2 Q$

Cain, Elizabeth Hope, 25, 2V

Campbell, Robert M., 3M

Campos-Delgado, Daniel U., IR

Canas, Liane S., OG

Candemir, Sema, 1E

Cao, Qing, OV

Caoili, Elaine M., 1V, 1Y, 29, 2B

Carin, Lawrence, $1 \mathrm{M}$

Cash, David M., OG

Casti, Paola, 2P

Catani, Juliana H., 2P

Cha, Kenny H., OQ, 1V, 1Y, 20, 21, 29, 2B

Chakraborty, Jayasree, 1N, 28, 2N

Chan, Chun-Wai, $3 Q$

Chan, Heang-Ping, $0 Q, 1 \mathrm{H}, 1 \mathrm{~V}, 1 \mathrm{Y}, 20,21,29,2 \mathrm{~B}$, 31

Chaovalitwongse, W. A., IF

Chechani, Shubham, OW

Chellamuthu, Karthik, 3B

Chen, Bo, 17

Chen, Fuyao, ON

Chen, Jerry, 2D

Chen, Kewei, ol

Chen, Wensheng, 17

Cheng, Bastian, $2 \mathrm{~F}$

Chevalier, Margarita, $2 \mathrm{~J}$

Chi, Pai-Chun Melinda, 10

Chin, J. L., 15

Chirra, Prathyush, $\mathrm{OB}$

Cho, Hyoun, 05

Chockanathan, Udaysankar, $\mathrm{OZ}$

Chong, Jo Woon, 12

Christie, Jason D., 3K

Christodouleas, John, 2D

Chughtai, Aamer, $1 \mathrm{H}$

Chung, Kaman, 2J

Cohan, Richard H., 1V, 1Y, 29, 2B

Cohen, Eric, $2 S$

Court, Laurence E., 10

Cresson, Thierry, OL, 3E

Crijns, T., 1D

Dammak, Salma, 03

Danala, Gopichandh, OP, 2K, 2L, 2O, 3N 
Davatzikos, Christos, 09, 2S

Davenport, Elizabeth, $\mathrm{OE}, \mathrm{OF}$

Dawant, Benoit M., ON

de Barros, Nestor, 2P

De Guise, Jacques, OL, 3E

de Oliveira, Helder C. R., 2P

de With, Peter H. N., OH, $3 \mathrm{O}$

Dellalana, Laura E., ON

de-Torres, Juan P., OM

Dhara, Ashis Kumar, 35

di Natale, Corrado, 2P

Dialani, Vandana, 2T

Dicente Cid, Yashin, 3A

Dighe, Manjiri, $1 \mathrm{X}$

Dijkstra, Jouke, OV

Do, Richard K. G., 1N, 28

Dong, Enqing, 14

Dormer, James D., 30

Drukker, Karen, OA, 3U

DSouza, Adora M., $0 Z$

Du, Yue, 2 L

Edwards, Alexandra, OA, 3U

Eguchi, Kenji, OX, 1G, 3D

Ellmore, Timothy M., OJ

Elmaraghy, Charles, IA

El-Zein, Randa, $3 Q$

Emaminejad, Nastaran, 1L, 3W

Enquobahrie, Andinet, $1 \mathrm{U}$

Erickson, Bradley J., 2G

Essig, Garth, 1A

Fang, Mengjie, 14

Fang, Yuan, $3 \mathrm{H}$

Fei, Baowei, 30

Fetita, Catalin, 05

Foy, Joseph J., $1 \mathrm{~K}$

Francis, Kathleen N., 3T

Fricke, Stanley Thomas, 3H

Fujita, Hiroshi, 2C, $2 \mathrm{U}$

Fukui, Tatsumasa, 2H

Fuller, Clifton D., 10

Furst, Jacob D., 38, 39

Gaber, M. Waleed, 3T

Gaed, M., 15

Gandikota, Dhanuj, 2B

Garg, Mandeep, 35

Gass, Tobias, 19

Gavrielides, Marios A., 3H

George, Jose, 11

Ger, Rachel B., 10

Gerritse, E. J., 1D

Ghafoorian, M., 1D

Gharaibeh, Yazan, $2 Y$

Ghate, Sujata V., 07, 2V

Giancardo, Luca, OJ

Gibson, E., 15

Giger, Maryellen L., OA, OS, OT, 1K, 2M, 3Q, 3U

Gillies, Robert James, 3P, 3S

Goerke, L., 1D

Goff, David L., 10

Goldgof, Dmitry B., 3P
Golsari, Amir, 2F

Gomez, J. A., 15

Gönen, Mithat, 1N

Gonzaga, Adilson, 2P

Gonzalez, Jessica, OM

Gordon, Marshall N., 1Y, 29

Greenspan, Hayit, 04, 06

Gregori, Johannes, 2F

Gremillet, Frederic, 3G

Grimm, Lars J., 07, OR, 2R, 2V, 2W

Gubern-Merida, Albert, 2J

Guo, Yanhui, 2Q

Gurcan, Metin N., 1A

Guzzo, Thomas J., 2D

Haas, Benjamin, 19

Hadjiiski, Lubomir M., 0Q, 1H, 1V, 1Y, 20, 21, 29, 2B, 3I

Hahn, Horst K., 19

Halicek, Martin, 30

Hall, Lawrence O., 3P

Han, Guanghui, 33

Hanaoka, Shouhei, IP

Hänsch, Annika, 19

Hara, Takeshi, 2C

Harada, Masafumi, 3D

Harowicz, Michael R., 07, 2W, 2X

Hashimoto, Noriaki, 34, 36

Hayashi, Naoto, $1 \mathrm{P}$

Hayashi, Tatsuro, 2H

Haynor, D. R., $1 \mathrm{~F}$

Heidari, Morteza, OP, 2K, 2L, 3F

Helvie, Mark A., OQ, 21, 31

Henschke, Claudia I., OM, 24

Heo, Changyong, 3L

Hermans, John, 30

Higuchi, Shunichi, $2 \mathrm{U}$

Hino, Koki, 3J

Hirano, Yasushi, 34, 36

Hironaka, Toru, 18, 3R

Ho, Arthur, 31

Hoffman, John, 1L, 3W

Hollingsworth, Alan B., 3N

Homma, Hirotoshi, 02

Hou, Rui, OR, 1M, 2R

Houghton, A. M., IF

Howell, Rebecca M., 10

Hsieh, Meng-Kang, 2S

Hsieh, Scott, 2T

Hu, Zhihong, 11

Huang, Shan, 17, 33

Huynh, Benjamin, 2M

Hwang, E. Shelley, OR, 2R, 2W

Iftekharuddin, K. M., OD

linuma, Gen, 27

Ishii, G., IG

Itoh, Hayato, 16

Iwano, Shingo, 02

Jagadale, Basavaraj N., 3M

Jagasia, Madan H., ON

Jago, James, $1 Z$ 
Jahani, Nariman, $2 S$

JanowCzyk, Andrew, OC

Janssen, D., 1D

Jarnagin, William R., 28

Jenuwine, Natalia M., 39

Jin, Hyeongmin, 3L

Jnawali, Kamal, $1 \mathrm{C}$

Jo, Javier, IR

Jones, Derek, OE, OF

Juarez-Chambi, Ronald M., IR

Kadoury, Samuel, 3G

Kalia, Sunil, 00

Kalloniatis, Michael, 31

Kalra, Naveen, 35

Kamali, Arash, 0J

Kaneko, Masahiro, OX, 1G, 3D

Karargyros, Alex, 1T

Karssemeijer, Nico, 2J

Karzcmar, Gregory, $3 \mathrm{U}$

Kato, Katsuya, 3J

Katsumata, Akitoshi, $2 \mathrm{H}$

Kawata, Yoshiki, OX, 1G, 27, 3D, 3J

Kazerooni, Ella, $1 \mathrm{H}$

Keating, Robert, $1 \mathrm{U}$

Kelley, Mireille, OE, OF

Kern, S., 1D

Khandelwal, Niranjan, 35

Kharazmi, Pegah, $0 \mathrm{O}$

Khilari, Rupal, 3C

Khokhar, Ashfaq, 3V

Kido, Shoji, 34, 36, 3R

Kim, Connie E., 07

Kim, Grace H., 1L, 3W

Kim, Hak Gu, 22

Kim, Jong Hyo, 3L

Kim, Seong Tae, 22

Kim, Young-Wouk, 05

Kinahan, P. E., $1 F$

King, Lauren $M$., $3 Q$

King, Lorraine M., OR, 2R

Kinoshita, Toshibumi, 2E

Kishimoto, Takumi, 3J

Kitasaka, Takayuki, 02

Kitslaar, Pieter H., OV

Klang, Eyal, 06

Klein, Jan, 19, 2F

Klimstra, David S., IN

Kline, Timothy L., 2G

Kobayashi, Kaoru, 2H

Kohli, Marc, 1 E

Kojima, Takuya, 2C

Kolluru, Chaitanya, $2 Y$

Konen, Eli, 04

Kontos, Despina, 25

Korfiatis, Panagiotis, $2 \mathrm{G}$

Kudavelly, Srinivas, IW

Kudo, Shin-ei, 16

Kumar, Abhishek, 35

Kuremoto, Takashi, 34

Kusumoto, Masahiko, OX, 1G, 3D
Kut, Carmen, IR

LaBarre, Victoria M., 38

Lampe, P. D., IF

Lan, Li, OS, 3Q

Langenhuizen, P. P. J. H., $\mathrm{OH}$

Lederer, David J., 3K

Lee, Hakmin, 22

Lee, Hannah J., 10

Lee, John H., OS

Lee, Juhun, 08

Lee, Tim K., $0 \mathrm{O}$

Leenstra, S., $\mathrm{OH}$

Legare, Philippe, $3 G$

Legters, M. J. W., OH

Lehman, Constance D., 2T

Lelieveldt, Boudewijn P. F., OV

Leo, Patrick, OB

Lepore, Natasha, $1 \mathrm{U}$

Levenkova, Anastasia, 31

Li, Heng, 10

Li, Hui, OS, OT, IK, 3Q

$\mathrm{Li}$, Jing, Ol

Li, Lihong C., 17

Li, Qin, 3H

Li, Songfeng, 31

Li, Xingde, IR

Liang, Zhengrong, 17, 2A

Liao, Guobin, 13, 26

Linguraru, Marius George, 1U, 12

Lippert, Fiona, $2 \mathrm{~F}$

Liu, Hong, 2O, 3N

Liv, Jiamin, 3B

Liu, Junchi, 36

Liu, Shuang, OM, 24

Liv, Xiabi, 33

Liv, Xiaonan, 01

Liu, Xiaoqi, 13, 26

Liu, Zhenyu, 14

Lo, Joseph Y., OR, 1M, 2R

Lu, Hong, 35

Lu, Hongbing, 2A

LU, Le, 3B

LU, Yao, 2Q, 31

Lui, Harvey, 00

Luo, Yahong, 23

Luo, Zhuo, 13

Lure, Fleming, 0 l

Ly, Angelica, 31

Ma, Ling, 30

Mabu, Shingo, 34

Mackey, Lester, 2T

Mackin, Dennis S., 10

MacMahon, Heber, 36

Madabhushi, Anant, OB, OC

Mahesh, Sunny N., 39

Makkinejad, Nazanin, 3V

Maklad, Ahmed S., 27

Maldjian, Joseph, OE, OF

Maley, Carlo C., OR, 2R

Mamtani, Ronac, 2D 
Manniesing, Rashindra, 1B, 1D, 1Q

Mansoor, Awais, $1 \mathrm{Z}$

Marks, Jeffrey R., OR, 2R

Martinelli, Eugenio, 2P

Mashhadi, Najmeh, 2L

Mastromanolis, Manuel, $1 \mathrm{U}$

Matsuhiro, Mikio, 27, 3D, 3J, 3R

Matsumoto, Y., $1 \mathrm{G}$

Matsuyama, Eri, 2E

Mattonen, Sarah, 03

Mazurowski, Maciej A., 07, OR, 0U, 25, 2R, 2V, 2W,

$$
\text { 2X }
$$

McDonough, Joseph, 3M

McKenney, Sarah, 3H

McNitt-Gray, Michael F., 1L, 3W

Mead, Simon, OG

Meijs, Midas, $1 Q$

Melo, Carlos F. E., 2P

Mencattini, Arianna, 2P

Mendel, Kayla R., OS, OT, 1K, 3Q

Midya, Abhishek, 1N, 28, 2N

Miki, Soichiro, IP

Mirniaharikandehei, Seyedehnafiseh, 3F

Misawa, Masashi, 16

Mitta, Prerana, $1 \mathrm{~K}$

Moberly, Aaron C., 1A

Modat, Marc, OG

Mohamed, Aly A., 23

Mol, J., 1D

Molteni, Erika, OG

Montillo, Albert, OE, OF

Morgas, Tomasz, 19

Mori, Kensaku, 02, 16

Mori, Masaki, 02

Mori, Yuichi, 16

Morita, Takako, $2 \mathrm{U}$

Moros, Eduardo G., 3P

Moussa, M., 15

Mu, Wei, 35

Mukhopadhyay, Sudipta, 35

Müller, Henning, 3A

Muramatsu, Chisako, $2 \mathrm{U}$

Murugesan, Gowtham Krishnan, OE, OF

Nakano, Yasutaka, 3D

Nandi, Debashis, 35

Näppi, Janne J., 18, 3R

Narayan, Nikhil S., $1 \mathrm{~W}$

Nasirudina, Radin A., 3R

Natori, Hiroshi, 02

Negahdar, Mohammadreza, 1J

Niki, Noboru, OX, 1G, 27, 3D, 3J

Nishikawa, Robert M., 08

Nomura, Yukihiro, $1 \mathrm{P}$

Nowosatka, Lauren R., 1K

Ocasio, Laura, OJ

Oda, Hirohisa, 02

Oda, Masahiro, 02, 16

Odhner, Dewey, 2D

Oh, Albert, $1 \mathrm{U}$

Ohmatsu, H., $1 \mathrm{G}$
Ohmura, Tomomi, 2E

Oiwa, Mikinao, 2U

Okada, Kazunori, 3C

Olory Agomma, R., 3E

Ourselin, Sébastien, OG

Pak, Linda M., 28

Palma, David, 03

Paniagua, Beatriz, $1 \mathrm{U}$

Pantalone, Lauren, 2S

Papaioannou, John, OA, 3U

Paramagul, Chintana, 1Y, 2B

Parent, Stefan, OL

Patel, Ajay, 1B

Patel, Alpen A., 1C

Patel, Bhavika, 2K

Patterson, Genevieve, 2T

Patwardhan, Kedar A., OW, IW

Paul, Rahul, 3P

Pautler, S., 15

Pearce, Melanie, 3N

Peck, Diondra, 2T

Pednekar, Gargi, 3K

Pereira, Carina, $1 \mathrm{X}$

Petrick, Nicholas, 3H

Pham, Dzung L., 21

Pickhardt, Perry J., 2A

Pierce, L. A., $1 F$

Pineda, Fred, 3U

Pipavath, S. N., IF

Platon, Alexandra, 3A

Poletti, Pierre-Alexandre, 3A

Pomeroy, Marc, 2A

Porras, Antonio R., $1 \mathrm{U}$

Powers, Alexander, OE, OF

Prabhu, David, $2 Y$

Prokop, Mathias, 30

Puchin, Juris, 3C

Pulvirenti, Alessandra, $1 \mathrm{~N}$

Puonti, Oula, 3A

Purysko, Andrei, OB

Qi, Jin, 3S

Qian, Wei, 20

Qin, Genggeng, 2Q

Qiu, Yuchen, OP, 2L

Quinones-Hinojosa, Alfredo, 1R

Qutub, Amina A., 3T

Rabidas, Rinku, 2N

Raicu, Daniela S., 38, 39

Ramachandran S., Sindhu, 11

Ramaraju, G. A., IW

Rao, Navalgund, $1 C$

Rastinehad, Ardeshir R., OB

Rathore, Saima, 09

Ravichandran, Kavya, OC

Reeves, Anthony P., OM, 24

Reidy, Diane L., $1 \mathrm{~N}$

Reilly, Carolyn M., 30

Remedios, Samuel, 21

Ren, Yinhao, 1M, 2R

Restivo, Michaela D., 3K 
Riascos-Castaneda, Roy, OJ

Richter, Caleb D., 0Q, 20, 21

Rico-Jimenez, Jesus, IR

Ro, Yong Man, 22

Rodriguez-Ruiz, Alejandro, 2J

Rogers, Gary F., $1 \mathrm{U}$

Rohde, Gustavo K., 08

Rosen, Mark, OB

Roth, Holger R., 02

Roubidoux, Marilyn A., 3l

Roy, Snehashis, 21

Rozycki, Martin, 09

Sadda, SriniVas R., 11

Sadhu, Anup, 2N

Saghafi, Behrouz, OE, OF

Saha, Ashirbani, 07, 0U, 25, 2V, 2W, 2X

Samala, Ravi K., 0Q, 1V, 20, 21, 29, 31

Sato, Daisuke, $1 \mathrm{P}$

Sauter, Jake N., 38

Schabath, Matthew, 35

Schiess, Mya C., OJ

Schifitto, Giovanni, $0 Z$

Schnabel, Julia A., 02

Schreibmann, Eduard, 30

Schwier, Michael, 19

Sechopoulos, loannis, 2J

Senan, Suresh, 03

Senaras, Caglar, 1A

Shafiq-ul-Hassan, Muhammad, 3P

Shboul, Z., OD

Sheth, Deepa, OT

Shi, Bibo, OR, 1M, 2R

Shi, Yanjie, 14

Shimada, Mitsuo, 27

Shimoda, Shinji, 2H

Shukla, Gaurav, 09

Simpson, Amber L., 1N, 28

Sivanandan, Srinivasan, IW

Sivaramakrishnan, R., 1E

Skaria, Shibon, 11

Soetemans, D., 15

Sonnenblick, Emily B., 24

Sowmya, Arcot, 31

Stafford, R. Jason, 10

Standvoss, K., 1D

Stitzel, Joel, OE, OF

Stoug, Rebecca G., 3N

Suescun, Jessika, 0J

Sugiura, Toshihiko, OX

Summers, Ronald M., 3B

Sun, Hongliu, $1 \mathrm{H}$

Sun, Yingshi, 14

Suresh, Rahul, OW

Suzuki, Hidenobu, 0X, 27, 3D, 3J

Suzuki, Kenji, 36, 3V

Syeda-Mahmood, Tanveer, $1 \mathrm{~J}, 1 \mathrm{~T}$

Syrgkanis, Vasilis, 2T

Tabei, Fatemehsadat, 12

Tabrizi, Pooneh R., 12

Tajbakhsh, Nima, 3V
Taj-Schaal, Nazhat, $1 \mathrm{~A}$

Takabatake, Hirotsugu, 02

Takahashi, Noriyuki, 2E

Takayama, Ryosuke, 2C

Takenaga, Tomomi, 1P

Tan, York-Kiat, OK

Tanabe, Nobuhiro, OX

Tang, Tien T., 3T

Tang, Zhenchao, 14

Tarando, Sebastián Roberto, 05

Tayob, Nabihah, 3Q

Teknos, Theodoros, $1 \mathrm{~A}$

Teuwen, Jonas, 2J

Thammasorn, P., IF

Thoma, George, $1 \mathrm{E}$

Thomalla, Göłz, 2F

Tian, Jie, 14

Tkaczyk, Eric R., ON

Tong, Yubing, 2D, 3K, 3M

Torigian, Drew A., 2D, 3K, 3M

Toyoshima, Hideto, 2E

Tsering, Deki, $1 \mathrm{U}$

Tsuchida, Takaaki, 1G, 3D

Tsuji, Daisuke, 3D

Tu, Liyun, $1 \mathrm{U}$

Tunali, llke, 35

Udupa, Jayaram K., 2D, 3K, 3M

Ukwatta, Eranga, $2 Z$

Urban, Jillian, $\mathrm{OE}, \mathrm{OF}$

V. V., Varun, 11

van de Vooren, D., ID

van den Heuvel, T. L. A., ID

van der Putten, Joost, 30

van der Sommen, Fons, 30

Van Leemput, Koen, 3A

van Niedek, T., 1D

van Vugt, J., ID

Vazquez, Carlos, OL, 3E

Veale, Tom, $0 G$

Venigalla, Sriram, 2D

Verheul, H. B., $\mathrm{OH}$

Vidyaratne, L., OD

Vieira, Marcelo A. C., 2P

Viswanath, Satish, OB

Wagner, Ben, OE, OF

Wahi-Anwar, Muhammad, 1L, 3W

Walsh, Ruth, 07

Wang, Chengliang, 13, 26

Wang, Chuang, 2D, 3K

Wang, Huafeng, 17

Wang, Jianing, ON

Wang, Shou, 14

Wang, Song, 2C

Wang, Yunzhi, 2O, 3F

Wang, Z. Jane, 00

Wang, Ziyuan, 11

Ward, Aaron D., 03, 15

Watari, Chinatsu, 3R

Watts, Nicolas, $3 G$

Wegmayr, Viktor, $1 \mathrm{~S}$ 
Wei, Jun, 1H, 2B, 3I

Wei, Qi, $1 M$

Wei, Xinzhou, 17

Weidman, David, 0 I

Weiler, Florian, 2F

Weinstein, Susan P., $2 S$

Weizer, Alon Z., 1V, 1Y, 29, 2B

White, James A., $2 Z$

Whitlow, Christopher, OE, OF

Whitman, Gary, 3Q

Whitney, Heather M., OA

Wilson, David L., $2 Y$

Wismüller, Axel, $0 Z$

Wolf, Matthias, 37

Wu, Caiyun, 3K, 3M

Wu, Hao, $2 Y$

Wu, Jimmy, 2T

Wu, Shandong, 23

Wu, Teresa, OI, 2K

Wu, W., $1 \mathrm{~F}$

XU, Shuoyu, OK

Xue, Zhiyun, $1 \mathrm{E}$

Yamada, Kazuma, 2C

Yamashita, Rikiya, $1 \mathrm{~N}$

Yan, Zhennan, 37

Yankelevitz, David F., OM, 24

Yeung, Pak-Hei, OK

Yim, Michael, OB

Yoshida, Hiroyuki, 18, 3R

Yoshikawa, Takeharu, IP

Yua, Lianbo, 1A

Yvernault, Benjamin, OG

Zabihollahy, Fatemeh, $2 Z$

Zarafshani, Ali, 2O, 3F

Zargari Khuzani, Abolfazl, OP, 2L

Zarshenas, Amin, 3V

Zawaski, Janice A., 3T

Zhan, Yiqiang, 37

Zhang, Jun, OU, 25, 2V, 2W, $2 \mathrm{X}$

Zhang, Lifei, 10

Zhang, Xiaoyan, 14

Zhang, Yaqin, 2Q

Zhao, Liang, 37

Zhao, Xinming, 33

Zhao, Yanfeng, 33

Zhao, Yiyuan, 37

Zheng, Bin, OP, 2K, 2L, 2O, 3F, 3N

Zheng, Jian, 28

Zhou, Bolei, 2T

Zhou, Chuan, 1H, 2B, 3l

Zhou, Chunwu, 33

Zhou, Shouhao, 10

Zhou, Xiangrong, 2C

Zhou, Xinxin, 2C

Zhou, Yuanpin, 2Q

Zhu, Zhe, OU, 25, 2V, 2W, 2X

Zinger, Svitlana, $\mathrm{OH}, 3 \mathrm{O}$

Zuley, Margarita L., 23

Zulueta, Javier, OM

xviii

Proc. of SPIE Vol. 10575 1057501-18 


\title{
Conference Committee
}

\author{
Symposium Chairs
}

Leonard Berliner, Weill Cornell Medical College (United States) and New York Presbyterian - Brooklyn Methodist Hospital (United States) Ronald M. Summers, National Institutes of Health (United States)

Conference Chairs

Nicholas Petrick, U.S. Food and Drug Administration (United States)

Kensaku Mori, Nagoya University (Japan)

Conference Program Committee

Samuel G. Armato III, The University of Chicago (United States)

Susan M. Astley, The University of Manchester (United Kingdom)

Stephen Aylward, Kitware, Inc. (United States)

Matthew S. Brown, University of California, Los Angeles (United States)

Heang-Ping Chan, University of Michigan (United States)

Weijie Chen, U.S. Food and Drug Administration (United States)

Marleen de Bruijne, Erasmus MC (Netherlands)

Thomas M. Deserno, Technische Universität Braunschweig (Germany)

Karen Drukker, The University of Chicago (United States)

Catalin Fetita, Télécom SudParis (France)

Hiroshi Fujita, Gifu University School of Medicine (Japan)

Maryellen L. Giger, The University of Chicago (United States)

Hayit Greenspan, Tel Aviv University (Israel)

Lubomir M. Hadjiiski, University of Michigan (United States)

Horst Karl Hahn, Fraunhofer MEVIS (Germany) and Jacobs University

Bremen (Germany)

Khan M. Iftekharuddin, Old Dominion University (United States)

Nico Karssemeijer, Radboud University Nijmegen Medical Center (Netherlands)

JongHyo Kim, Seoul National University Hospital (Korea, Republic of)

Marius George Linguraru, Children's National Medical Center (United States)

Maciej A. Mazurowski, Duke University (United States)

Fabrice Meriaudeau, University Teknologi Petronas (Malaysia)

Janne J. Näppi, Massachusetts General Hospital (United States) and Harvard Medical School (United States)

Noboru Niki, University of Tokushima (Japan)

Carol L. Novak, Siemens Healthineers (United States)

Clarisa I. Sánchez, Radboud University Medical Center (Netherlands) 
Ronald M. Summers, National Institutes of Health (United States)

Kenji Suzuki, Illinois Institute of Technology (United States)

Georgia D. Tourassi, Oak Ridge National Laboratory (United States)

Bram van Ginneken, Radboud University Nijmegen Medical Center (Netherlands)

Rafael Wiemker, Philips Research (Germany)

Axel Wismüller, University of Rochester Medical Center (United States)

Xiaofeng Yang, Emory University (United States)

Hiroyuki Yoshida, Massachusetts General Hospital (United States) and Harvard Medical School (United States)

\section{Session Chairs}

1 Lung I and Liver

Catalin Fetita, Télécom SudParis (France)

Weijie Chen, U.S. Food and Drug Administration (United States)

2 Radiomics

Maryellen L. Giger, The University of Chicago (United States)

Thomas Martin Deserno, Technische Universität Braunschweig (Germany)

3 Brain I

Hiroshi Fujita, Gifu University School of Medicine (Japan)

Baowei Fei, Emory University (United States)

$4 \quad$ Musculoskeletal and Skin

Karen Drukker, The University of Chicago (United States)

Carol L. Novak, Siemens Healthineers (United States)

5 Breast I

Heang-Ping Chan, University of Michigan Health System (United States)

Susan M. Astley, The University of Manchester (United Kingdom)

6 Cardiac, Vessels, and Novel Applications

Weijie Chen, U.S. Food and Drug Administration (United States)

Horst K. Hahn, Fraunhofer MEVIS (Germany) and Jacobs University Bremen (Germany)

7 PROSTATEx Lessons Learned and 2019 Challenge

Samuel G. Armato III, The University of Chicago (United States)

Nicholas A. Petrick, U.S. Food and Drug Administration (United States) 
8 Keynote and Eye

Kensaku Mori, Nagoya University (Japan)

Lubomir M. Hadjiiski, University of Michigan Health System

(United States)

9 Colon and Prostate

Janne J. Näppi, Massachusetts General Hospital (United States)

Hiroyuki Yoshida, Massachusetts General Hospital (United States)

10 Head and Neck

Hayit Greenspan, Tel Aviv University (Israel)

Xiaofeng Yang, Emory University (United States)

11 Lung II

Georgia D. Tourassi, Oak Ridge National Laboratory (United States)

Jong Hyo Kim, Seoul National University Hospital (Korea, Republic of)

12 Quantitative

Bram van Ginneken, Radboud University Medical Center

(Netherlands)

Marleen de Bruijne, Erasmus MC (Netherlands)

13 Brain II

Axel Wismüller, University of Rochester Medical Center (United States)

Kenji Suzuki, Illinois Institute of Technology (United States)

14 Other Organs

Marius George Linguraru, Children's National Medical Center (United States)

Ronald M. Summers, National Institutes of Health (United States)

15 Breast II

Nico Karssemeijer, Radboud University Medical Center (Netherlands)

Maciej A. Mazurowski, Duke University (United States) 
Proc. of SPIE Vol. 10575 1057501-22 Downloaded From: https://www.spiedigitallibrary.org/conference-proceedings-of-spie on 25 Apr 2023
Terms of Use: https://www.spiedigitallibrary.org/terms-of-use 


\section{Medical Imaging Award Recipients}

\section{Robert F. Wagner Best Student Paper Award}

Robert F. Wagner was an active scientist in the SPIE Medical Imaging meeting, starting with the first meeting in 1972 and continuing throughout his career. He ensured that the BRH, and subsequently the $\mathrm{CDRH}$, was a sponsor for the early and subsequent Medical Imaging meetings, helping to launch and ensure the historical success of the meeting. The Robert F. Wagner All-Conference Best Student Paper Award (established 2014) is acknowledgment of his many important contributions to the Medical Imaging meeting and his many important advances to the field of medical imaging.

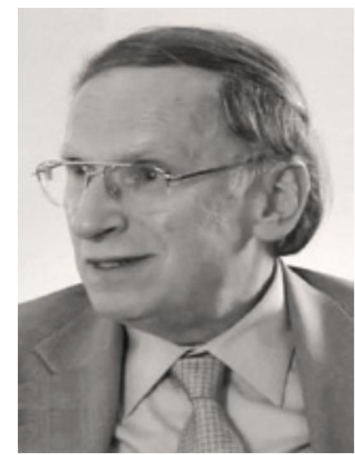

This award is co-sponsored by:

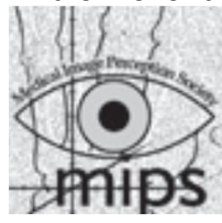

The Medical Image Perception Society

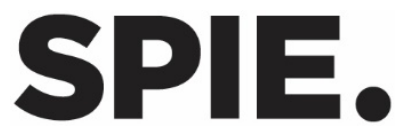

\section{Recipients:}

First Place: Dynamic beam filtering for miscentered patients (10573-29)

Andrew Mao, William Shyr, Grace J. Gang, J. Webster Stayman, Johns Hopkins Univ. (United States)

Second Place: Tumor margin classification of head and neck cancer using hyperspectral imaging and convolutional neural networks (10576-4)

Martin Halicek, Georgia Institute of Technology (United States) and Augusta Univ. (United States); James V. Little, Xu Wang, Emory Univ. School of Medicine (United States); Mihir Patel, Emory Univ. School of Medicine (United States) and The Winship Cancer Institute of Emory Univ. (United States); Christopher C. Griffith, Emory Univ. School of Medicine (United States); Amy Y. Chen, Emory Univ. School of Medicine (United States) and The Winship Cancer Institute of Emory Univ. (United States); Baowei Fei, Georgia Institute of Technology \& Emory Univ. (United States) and The Winship Cancer Institute of Emory Univ. (United States) 
Proc. of SPIE Vol. 10575 1057501-24 Downloaded From: https://www.spiedigitallibrary.org/conference-proceedings-of-spie on 25 Apr 2023
Terms of Use: https://www.spiedigitallibrary.org/terms-of-use 